\title{
The safety and efficacy of mini-percutaneous nephrolithotomy vs. retrograde intrarenal surgery for treatment of renal lithiasis in pelvic ectopic kidney: an exploratory pilot study
}

\author{
Junfeng $\mathbf{W u}$, Jun Shen \\ Department of Urology, Qiandongnan People's Hospital Affiliated to Guizhou Medical University, Kaili, China \\ Contributions: (I) Conception and design: All authors; (II) Administrative support: All authors; (III) Provision of study materials or patients: All \\ authors; (IV) Collection and assembly of data: All authors; (V) Data analysis and interpretation: All authors; (VI) Manuscript writing: All authors; (VII) \\ Final approval of manuscript: All authors. \\ Correspondence to: Jun Shen. Department of Urology, Affiliated Hospital of Guizhou Medical University, 28 Guiyi Street, Yunyan District, Guiyang, \\ China. Email: dgixbvykwqso@sina.com.
}

Background: To compare the safety and efficacy of mini-percutaneous nephrolithotomy (PCNL) and retrograde intrarenal surgery (RIRS) for the treatment of renal lithiasis in patients with pelvic ectopic kidney. Methods: From January 2015 to October 2017, mini-PCNL and RIRS were performed in ten patients diagnosed with lithiasis in pelvic ectopic kidneys, including three cases under laparoscopy-assisted miniPCNL. Patient demographics and perioperative characteristics (age, gender, BMI, side of pelvic kidney, stone size, stone number, stone location, special medical history, and ASA physical status classification), and operative and post-operative related details (operation time, hospital stay, blood loss, VAS, analgesic requirement, complications, and stone free outcome) were reviewed.

Results: Although the mean operation time of mini-PCNL (71.3 min) was shorter than RIRS (85.3 min), the mean operation time of laparoscopy assisted mini-PCNL (92 min) was longer than patients without laparoscopy-assisted mini-PCNL (55.8 min). However, the use of mini-PCNL allowed for larger lithiasis to be dealt with $(1.9 \mathrm{~cm}$ in laparoscopy assisted mini-PCNL and $2.4 \mathrm{~cm}$ in mini-PCNL without laparoscopyassist) compared with RIRS $(1.2 \mathrm{~cm})$. In addition, although the mean hospital-stay time, blood loss, and analgesic requirement of patients undergoing RIRS were less than those receiving mini-PCNL, the success rate of RIRS was only 50\% (3/6) in comparison to $100 \%(7 / 7)$ for mini-PCNL. Except for pain and urinary tract infection after the operation, there were no significant intraoperative and postoperative complications, and no residual lithiasis were seen in any patient.

Conclusions: Although RIRS was less time-consuming and invasive, mini-PCNL can deal with the bigger lithiasis and more complex situations with a higher success rate. Both mini-PCNL and RIRS are feasible and safe treatments for pelvic ectopic kidney lithiasis with each carrying unique advantages. Hence in practice, an appropriate individualized treatment should be selected depending on patient characteristics.

Keywords: Pelvic ectopic kidney; mini-percutaneous nephrolithotomy (mini-PCNL); retrograde intrarenal surgery (RIRS); laparoscopy-assisted; renal lithiasis

Submitted Jan 14, 2021. Accepted for publication Feb 10, 2021.

doi: $10.21037 / \mathrm{tau}-21-77$

View this article at: http://dx.doi.org/10.21037/tau-21-77 


\section{Introduction}

Pelvic ectopic kidney is the most common form of renal malformation with an incidence ranging from 1 in 2,200 to 1 in 3,000 (1). The condition results from a failure of ascent to the normal anatomic location in the renal fossa during kidney development, leaving the kidney below the pelvic brim (2). Pelvic ectopic kidney is often accompanied by renal malrotation and a high insertion of the ureter, leading to the inadequate evacuation of urine and formation of renal lithiasis (3). Furthermore, this abnormal orientation creates altered spatial relations with adjacent organs (as the kidney lies anterior to the sacrum, caudal to the aortic bifurcation, and posterior to the peritoneum), abnormal calyceal orientation, and anomalous vascular patterns (deriving blood supply from iliac vessels or distal aorta). This makes the active treatment of lithiasis in the pelvic ectopic kidney a great challenge for surgeons (4).

Several approaches, including extracorporeal shock wave lithotripsy (SWL), laparoscopic pyelolithotomy, retrograde intrarenal surgery (RIRS), percutaneous nephrolithotomy (PCNL), and open surgery are available for the treatment of renal lithiasis in anatomically normal kidneys. However, there are no clear guidelines for the treatment of lithiasis in pelvic ectopic kidney. PCNL and RIRS are the most widely used treatments for upper urinary tract lithiasis and each holds unique advantages. In this study, we applied our exploratory pilot experiences of mini-PCNL and RIRS for the treatment of lithiasis in the pelvic ectopic kidney and compared the safety and efficacy of these methods. The results of this study may help clarify treatment options in the management of patients with lithiasis and pelvic ectopic kidney.

We present the following article in accordance with the MDAR reporting checklist (available at http://dx.doi. org/10.21037/tau-21-77).

\section{Methods}

An exploratory pilot study was undertaken in ten patients diagnosed with pelvic ectopic kidney lithiasis in our hospital between January 2015 and October 2017. The study conformed to the provisions of the Declaration of Helsinki (as revised in 2013). The study was approved by the medical ethics committee of the Qiandongnan People's Hospital Affiliated to Guizhou Medical University. All patients agreed to participate in this study and signed an informed consent form. Mini-PCNL and RIRS were performed in ten patients diagnosed with lithiasis in pelvic ectopic kidneys, including three cases under laparoscopy-assisted miniPCNL. The mean age and BMI of the six male and four female patients were 37.9 years (range, 27-66 years) and $21.8 \mathrm{~kg} / \mathrm{m}^{2}$ (range, $19.1-24.9 \mathrm{~kg} / \mathrm{m}^{2}$ ), respectively (Table 1 ). Pelvic ectopic kidneys were located on the left side in $70 \%$ (7/10) of patients and the final diagnosis was based on the results of computed tomography urography (CTU). The size, number, and location of stone are also summarized in Table 1. Two patients had a history of open surgery for pelvic ectopic kidney lithiasis, one patient had a horseshoe kidney, and another had scoliosis. The exclusion criteria for surgery included an American Society of Anesthesiologists (ASA) physical status more than grade III.

All cases were routinely examined with laboratory tests, including routine blood, blood biochemistry, blood coagulation function, routine urine, and urine culture. In addition, CTU, computed tomography angiography (CTA), and three-dimensional (3D) reconstruction were implemented as routine radiologic evaluations to determine the size, number, and location of the stone, and the organ position and vessel shape around the pelvic ectopic kidney (Figure 1).

All operations were performed under general anesthesia in the lithotomy position. A 6 Fr ureteral catheter was first introduced retrograde into the pelvic ectopic kidney under fluoroscopic guidance using a cystoscope. Patients were then adjusted to the supine position except for one patient who was placed in the lateral position because of scoliosis, in whom a roller pack was placed to lift the kidney closer to the anterior abdominal wall.

The target calyx was determined using preoperative CT (including CTU, CTA, and $3 \mathrm{D}$ reconstruction) and intraoperative ultrasound monitoring (Figure 2A,B). After ultrasound-guided puncturing of the target calyx (Figure 2C), a $0.89 \mathrm{~mm}$ U-shaped guidewire was used to reconfirm access for an $18 \mathrm{G}$ needle and the operative validity of the calyx (Figure 2D), and to guide the track dilatation. Multi-step dilatation was used, and a $20 \mathrm{Fr}$ sheath and Karl Storz 12 Fr nephroscope were used for the miniPCNL procedure. Lithiasis were fragmented by Holmium laser, and after extraction, a 6 Fr D-J stent was inserted into the ureteral lumen and a 16 or $18 \mathrm{Fr}$ nephrostomy tube was placed where the sheath was removed.

In the three patients undergoing laparoscopy-assisted mini-PCNL the laparoscope was used to observe whether the needle passed through other organs or major vessels, and to monitor the process of multi-step dilatation 
Table 1 Patient related details

\begin{tabular}{|c|c|c|c|}
\hline Variable & \multicolumn{2}{|c|}{ Mini-PCNL } & $\operatorname{RIRS}(n=3)$ \\
\hline Mean age [range], years & 33 [29-42] & 46 [27-67] & 32 [26-37] \\
\hline Gender (male/female) & $1 / 2$ & $3 / 1$ & $2 / 1$ \\
\hline Mean BMI [range], kg/m² & $19.8[18.8-21.6]$ & $22.5[20.1-23.9]$ & $22.8[21.1-24.9]$ \\
\hline Mean stone size [range], cm & $1.9[1.0-3.3]$ & $2.4[1.1-3.4]$ & $1.2[0.8-1.5]$ \\
\hline \multicolumn{4}{|l|}{ Stone number } \\
\hline Solitary & 2 & 3 & 2 \\
\hline Multiple & 1 & 1 & 1 \\
\hline Pelvis & 1 & 3 & 1 \\
\hline Lower calyx & 2 & 0 & 0 \\
\hline \multicolumn{4}{|l|}{ Special medical history } \\
\hline Open surgery & 1 & 1 & 0 \\
\hline Horseshoe kidney & 0 & 1 & 0 \\
\hline Scoliolosis & 0 & 1 & 0 \\
\hline \multicolumn{4}{|l|}{ ASA classification } \\
\hline Grade I & 3 & 2 & 3 \\
\hline
\end{tabular}

PCNL, mini-percutaneous nephrolithotomy; RIRS, retrograde intrarenal surgery.

(Figure 3A,B). Two laparoscopic holes were punched, and 12 and $5 \mathrm{~mm}$ trocars were placed below the umbilicus and on the left abdomen, respectively (Figure 3C). Once a successful puncture was established, multi-step dilatation was performed under reduced abdominal pressure from 15 to $10 \mathrm{mmHg}$ and irrigation solutions or urine were aspirated by the suction device at the same time as fragmenting the lithiasis. Finally, in addition to the indwelling $16 \mathrm{Fr}$ nephrostomy tube and 6 Fr D-J stent, a 14 Fr abdominal drainage tube was placed into the location of the $5 \mathrm{~mm}$ trocar.

A Cook 12 or 14 Fr ureteral access sheath was retrogradely placed along the guide wire in the lithotomy position for the RIRS procedure. Subsequently, an Olympus URF-V flexible ureteroscope with $8.4 \mathrm{Fr}$ tip diameter was inserted to inspect the pelvicalyceal system and identify the location of lithiasis. Lithotripsy was performed by a $200 \mu \mathrm{m}$ Holmium laser fiber with less than $20 \mathrm{~W}$ (energy level of
0.6 to $1.0 \mathrm{~J}$ and pulse rate of 10 to $20 \mathrm{~Hz}$ ), and the nitinol basket and grasper were used to retrieve the resultant stone fragments. Finally, the ureteroscope and ureteral access sheath were removed, and a 6 Fr D-J stent was retained.

Routine blood and biochemistry tests were performed the morning after surgery. The catheter was removed one day later, and the nephrostomy tube was removed when drainage was less than $50 \mathrm{~mL}$ every 24 hours. In patients undergoing laparoscopy-assisted procedures, the abdominal drainage tube was removed after bleeding or extravasation was less than $10 \mathrm{~mL}$ every 24 hours. All patients were scheduled to followup 4 weeks later, and the D-J stent was removed once lithiasis were not detected using X-ray or CT.

\section{Statistical analysis}

SPSS 21.0 software (SPSS Inc., Chicago, IL, USA) was used for statistical analysis. Measurement data conforming to a 

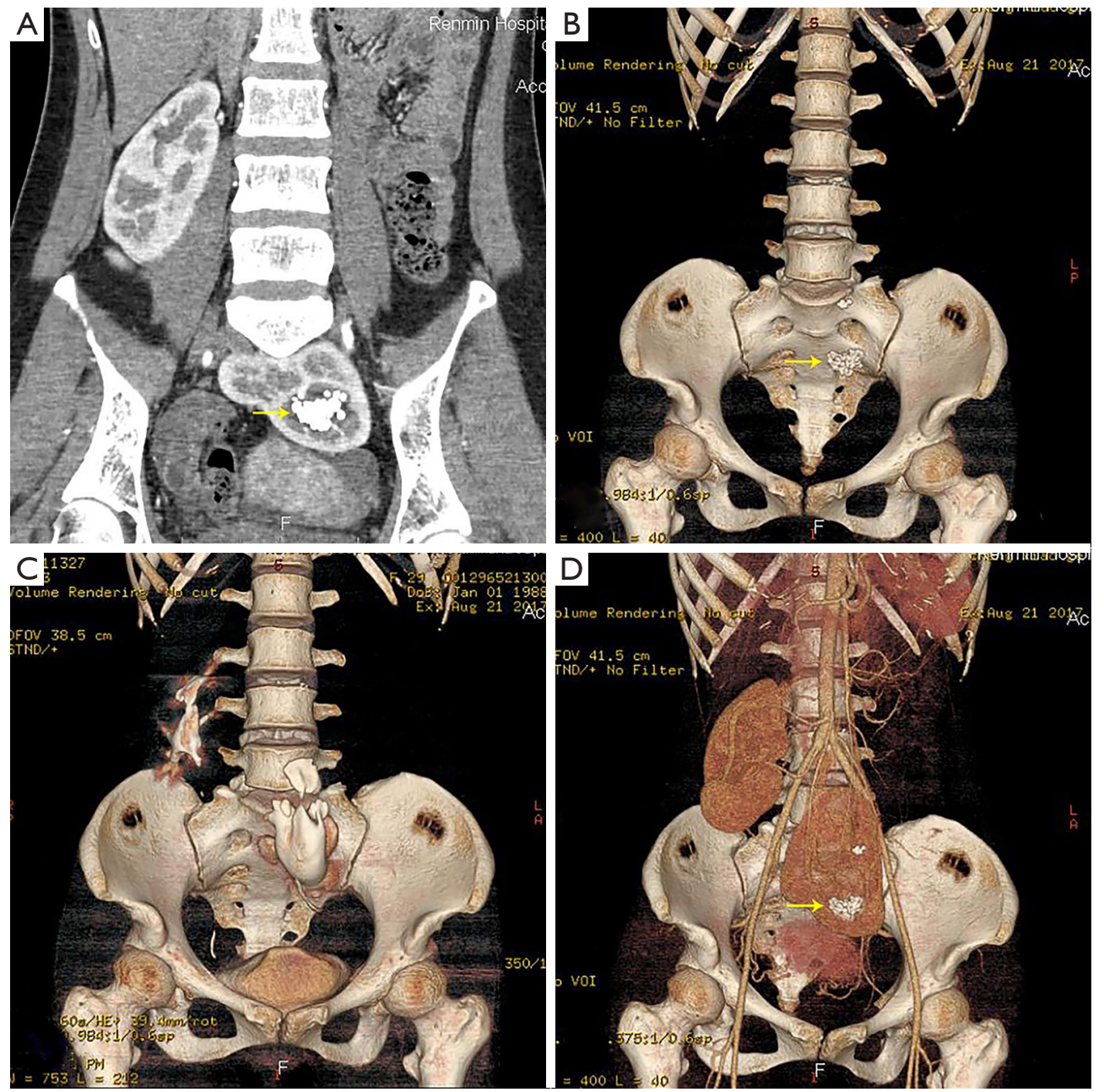

Figure 1 Radiologic evaluation using computed tomography urography (CTU), computed tomography angiography (CTA), and 3D reconstruction. (A) Image of a patient with multiple lithiasis within the left pelvic ectopic kidney; (B,C) multiple lithiasis and collecting system are shown in three-dimensional reconstruction of CTU; (D) vessel shape around the pelvic ectopic kidney in CTA. The yellow arrow indicates the location of the lithiasis.

normal distribution was described by mean \pm SD and an independent sample $t$-test was used.

\section{Results}

The demographic, perioperative characteristics, and operative and post-operative related results are presented in Tables 1 and 2. Six patients with lithiasis smaller than $2 \mathrm{~cm}$ were initially treated by RIRS, but $50 \%$ of these cases $(3 / 6)$ were unsuccessful due to failed insertion of the ureteral access sheath or abnormal calyceal structure hindering lithotripsy. These patients were then treated with mini-PCNL. Although two patients had multiple lithiasis, a single channel for mini-PCNL was used in seven patients because their lithiasis were located in a limited renal calyx or pelvis. The mean operation time of miniPCNL procedures $(71.3 \mathrm{~min})$ was shorter than RIRS (85.3 $\mathrm{min})$. Due to the time taken for laparoscopy preparation and observation, the mean operation time of laparoscopy-assisted mini-PCNL was $92 \mathrm{~min}$, which was longer than mini-PCNL without laparoscopy-assistance (55.8 $\mathrm{min}$ ) and RIRS (85.3 min). However, the use of miniPCNL with or without laparoscopy-assistance allowed us to deal with larger lithiasis $(1.9$ and $2.4 \mathrm{~cm}$ ) in comparison with RIRS (the largest $1.2 \mathrm{~cm}$ ).

The mean operative blood loss, post-operative VAS 

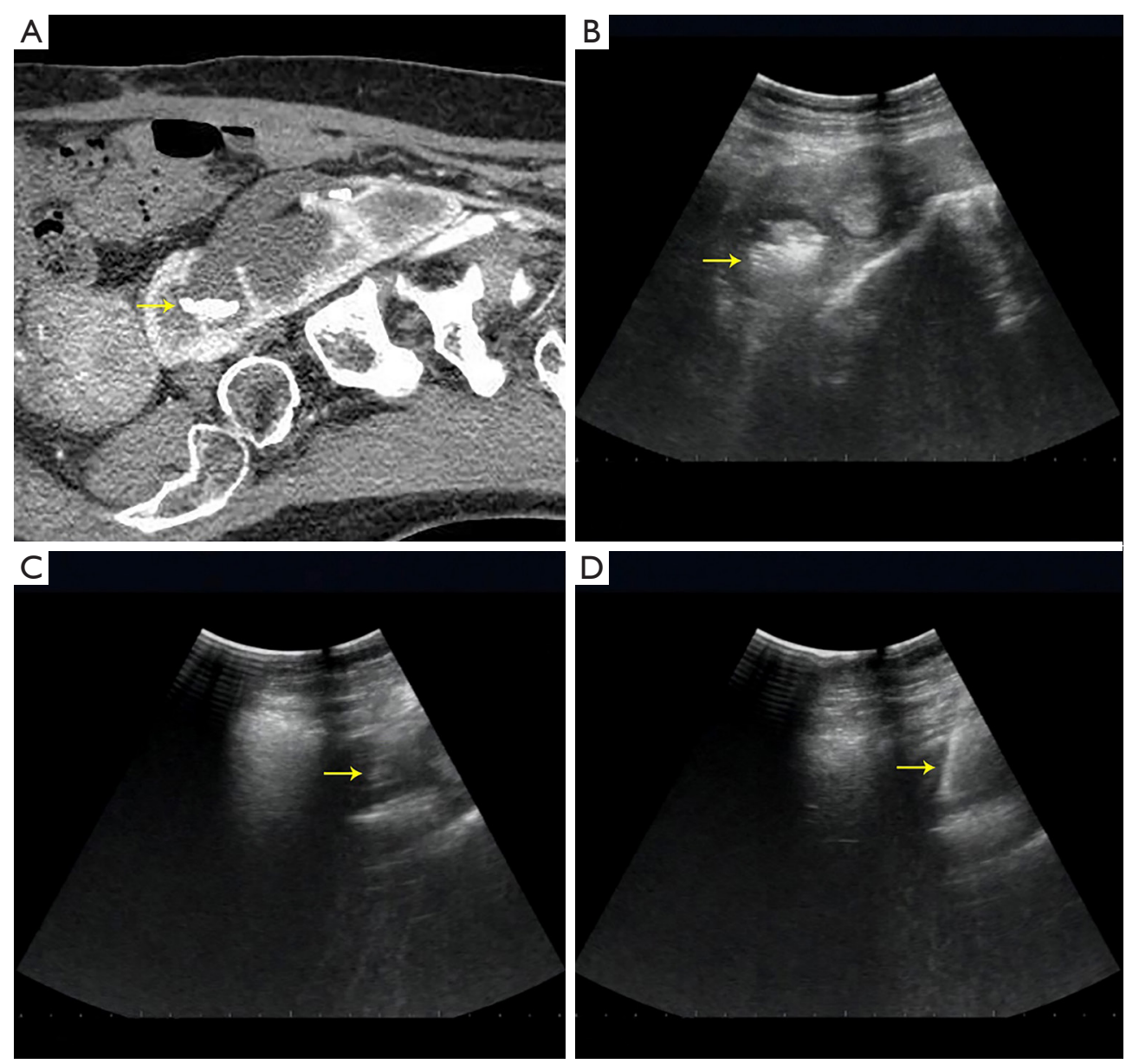

Figure 2 Preoperative computed tomography urography (CTU) and intraoperative ultrasound. (A,B) The images of lithiasis and collecting system using preoperative CTU and intraoperative ultrasound were matched; the yellow arrow indicates the location of the lithiasis; (C,D) the images of U-shaped guidewire in intraoperative ultrasound monitoring. The yellow arrow indicates the location of U-shaped guidewire.

score, and analgesic requirement of patients who underwent RIRS were less than both mini-PCNL with or without laparoscopy-assistance. Except for pain and urinary tract infection after the operation, no patient experienced severe intraoperative or postoperative complications including severe bleeding, sepsis, and urine leakage after nephrostomy tube removal. One patient had a fever because of a urinary tract infection after surgery, but the infection was controlled with routine antibiotics treatment. In addition, there were no instances of bowel injury or abdominal infection in patients who underwent laparoscopy-assisted procedures. The mean hospital stay time of RIRS (3 days) was less than mini-PCNL (5.1 days) and no residual lithiasis were found in any patients at the four-week follow-up and the D-J stent was removed successfully in all patients.

\section{Discussion}

Patients with pelvic ectopic kidney are prone to chronic obstruction and renal lithiasis due to the abnormal structure and architecture seen in this condition. This variation in anatomy, including anomalous vascular patterns and altered spatial relations with adjacent pelvic organs also presents difficult decisions for those performing nephrolithiasis treatment (3).

SWL is a first-line and established method for the treatment of renal lithiasis less than $2 \mathrm{~cm}$. SWL is also the least invasive of common treatment methods used to treat renal lithiasis and can take place without the need for hospitalization (5). However, the results of research on its use in treating patients with pelvic ectopic kidneys differ, 

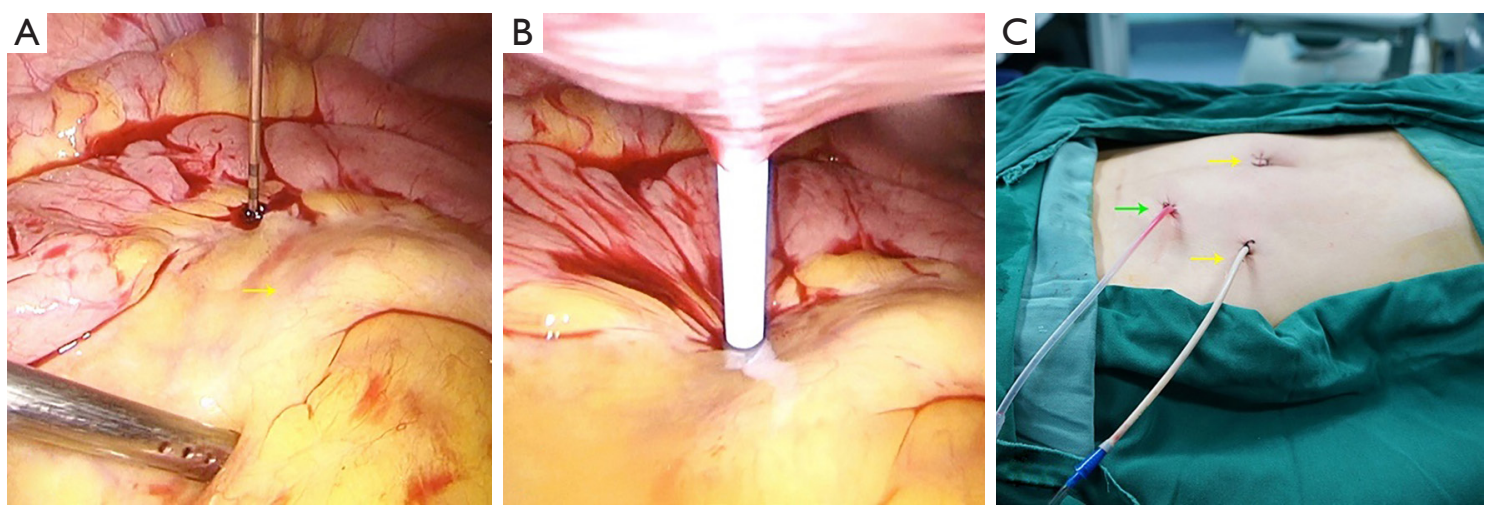

Figure 3 Intraoperative laparoscopic detection. (A,B) Monitoring needle and multi-step dilatation under laparoscopy assisted surgery; (C) nephrostomy tube and abdominal drainage tube placement. A 20 Fr sheath was placed in the location of the green arrow (nephrostomy tube) and a 12 and $5 \mathrm{~mm}$ trocar were placed in the location of the yellow arrow below the umbilicus and left abdomen (abdominal drainage tube), respectively.

Table 2 Operative and post-operative related details

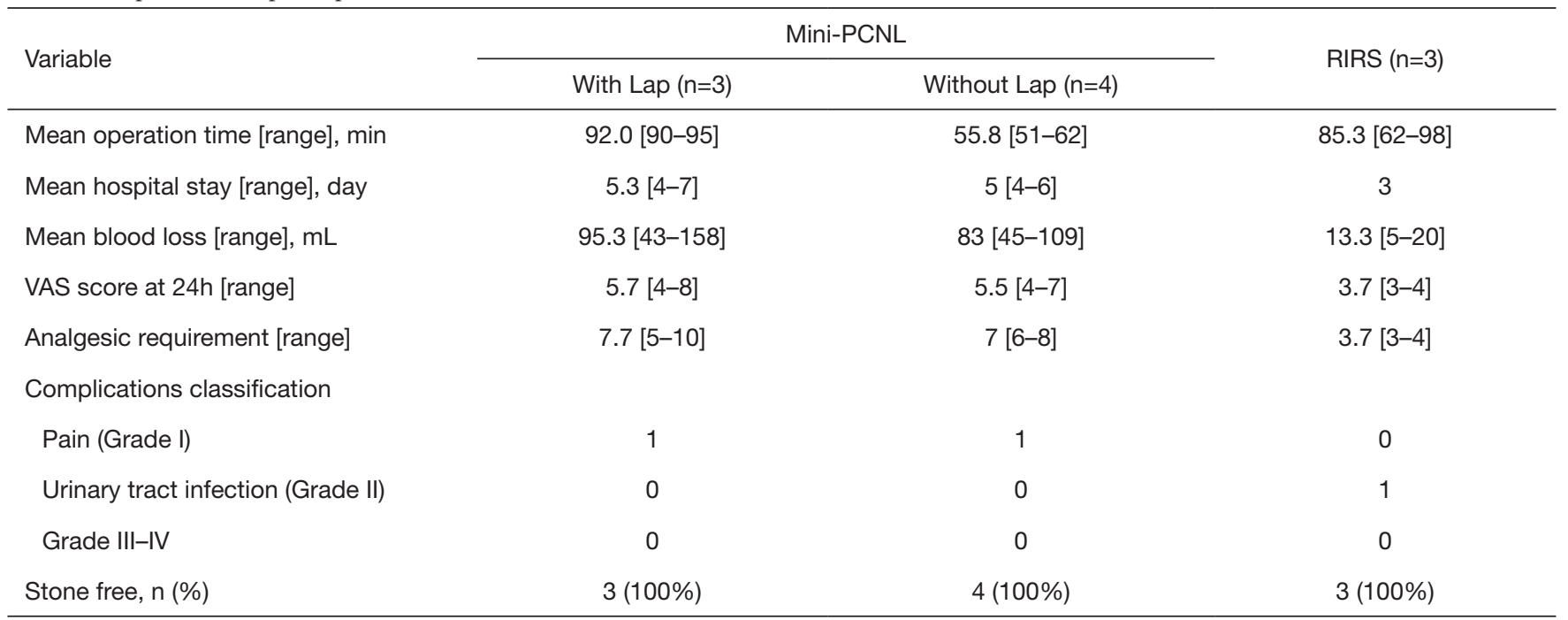

PCNL, mini-percutaneous nephrolithotomy; RIRS, retrograde intrarenal surgery.

with varying stone free rates, and some patients requiring additional remedies. A monotherapy study showed that $82 \%(9 / 11)$ of patients were stone free at the three months follow-up when SWL was used to treat urinary lithiasis in pelvic ectopic kidneys, although obstructive dural embolism was formed in two patients and only one was under an ancillary endourologic procedure (6). Demirkesen et al. (7) compared SWL outcomes in normal and abnormal upper urinary lithiasis, including eight patients with pelvic ectopic kidney, and found those with a normal kidney had higher stone free rates $(78 \%)$ than those with an abnormal kidney $(56 \%)$, and the rates of clinically insignificant residual stone fragments in normal and abnormal kidneys were $18.5 \%$ and $37 \%$, respectively. There are two main factors which limit the therapeutic effect of SWL in pelvic ectopic kidneys. Firstly, the bony pelvis and bowel cripple the effective of SWL. Secondly, the high insertion of the ureter and impaired pyeloureteral mobility by fibrous bands which surround the kidney forming abnormal drainage patterns hinders the expulsion of residual fragments which promotes infection and stone recurrence (8).

In comparison to PCNL, laparoscopic pyelolithotomy, and open surgery, RIRS has become an increasingly popular procedure to treat lithiasis due to its less invasive 
nature. Using a 7.5 Fr flexible ureteroscope, holmium laser lithotripsy, and nitinol baskets and graspers, six of eight patients with an average $1.4 \mathrm{~cm}$ stone burden in anomalous kidneys (including four patients with pelvic ectopic kidney), had complete clearance of the lithiasis, with seven being asymptomatic after the procedure (9). However, RIRS has its limitations in stone burden and cannot deal with the pathologic structure of the pelvic ectopic kidney which restricts the insertion of the ureteral access sheath and removal of residual fragments. In addition, RIRS carries the problem of infection. Although no significant statistical difference was found in the total complication rate between treatment with PCNL and RIRS for renal lithiasis, the longer operation time and higher intrarenal pressure of RIRS compared to PCNL increased the risk of sepsis (10). In our study, six patients with lithiasis less than $2 \mathrm{~cm}$ were initially treated by RIRS, but 50\% (3/6) failed because of a failure in the insertion of the ureteral access sheath because of the abnormal calyceal structure. The three unsuccessful cases then underwent a mini-PCNL procedure. Despite this, there were no significant intraoperative and postoperative complications in RSIS patients and no residual lithiasis in successful cases. Therefore, to improve the safety and stone clearance rate of RIRS in patients with pelvic ectopic kidney, we recommend urinary tract infection should be controlled preoperatively, the procedures should only be used to treat appropriately sized lithiasis and those easily accessed, and the effective application of surgical instruments such as nitinol baskets and graspers should be used.

PCNL is a popular and conventional surgical method widely used to treat patients with all types of upper urinary tract lithiasis in the orthotopic kidney, and has a low incidence of significant complications such as hemorrhage, sepsis, and injury of perirenal organs. However, there is dispute over the performance of PCNL on pelvic ectopic kidney lithiasis. Our experience in the treatment of pelvic ectopic kidney calculus with transabdominal mini-PCNL using ultrasound-guided puncture follows.

We use fluoroscopic or ultrasound guidance puncture methods. Although fluoroscopy has been the main tool to establish access in PCNL, its excessive use leads to an increase in the risk of radiation to patients and staff (11). The use of ultrasound-guided puncture in PCNL was first reported in the 1970s (12) and has gained increased recognition as an alternative to fluoroscopy because of its feasibility, safety, and efficacy. Ultrasound guidance not only has the advantage of low radiation exposure, it can also monitor the image of lithiasis and collecting systems in three-dimensional (3D) orientation during surgical procedures (13). Moreover, ultrasound guidance can image intervening structures between the skin and kidney and monitor bowel movement to avoid injury to the bowel during the puncture procedure. Despite this, there is a reluctance on behalf of some operators to use ultrasound as this requires significant training, and the images produced may be difficult to interpret.

In addition to using intraoperative ultrasound guidance, preoperative radiologic evaluation using CTU, CTA and 3D reconstructions also plays a crucial role during puncturing. A greater number of channels increases the risk of potential complications, hence the selection of the desired calyx is crucial. A desirable access should be designed according to the combined outcomes of intraoperative monitoring by ultrasound and preoperative radiologic evaluation of CTU and $3 \mathrm{D}$ reconstruction which provide important information regarding stone size, number, location, the structure of collecting systems, and the relationship between lithiasis and collecting system. In addition, CTA and 3D reconstruction can display anomalous vascular patterns around the pelvic ectopic kidney and provide assistance to reduce renal and perirenal vessel injury.

We also do not use transabdominal access as a routine method for PCNL. A roller pack is placed under the pelvic ectopic kidney to bring the target calyx closer to the anterior abdominal wall to facilitate access during PCNL. Furthermore, even though the operation time with laparoscopy-assisted PCNL is longer than other methods because of the procedure in laparoscopy preparation and observation, the operation procedures especially in puncture and dilatation became safer. With laparoscopicassistance, blood, urine and washing solution are aspirated by the suction device while fragmenting lithiasis, thus avoiding abdominal infection and irritation to the bowel by these irritants. However, laparoscopy-assisted PCNL has limitations in some patients who have a history of other disease and an unusual location of renal lithiasis.

Lastly, standard PCNL requires a larger tract, which may increase the risk of bleeding in a kidney with anomalous blood supply (14). Based on the comprehensive consideration of operation time and stone clearing efficiency, the $12 \mathrm{Fr}$ nephroscope matched with $20 \mathrm{Fr}$ channel is preferred in our clinical center. However, for some special cases we will also use negative pressure suction equipment matched with the standard channel or finer nephroscope with the smaller channel. In addition, there 
is also a risk of urinary leakage in tubeless PCNL which may cause ileus and morbidity (15). Therefore, the use of tubeless PCNL as a routine procedure is debatable.

Several recent reports discussing the treatment of pelvic ectopic kidney lithiasis by RIRS and PCNL, with or without laparoscopy-assistance, have expressed results similar to ours $(4,16,17)$. Nevertheless, these reports have not produced a systematic treatment strategy for the disease. Overall, we summarize guidelines for the treatment of lithiasis in the pelvic ectopic kidney according to our experiences as follows. (I) If the size of a solitary stone or total multiple lithiasis is less than $2 \mathrm{~cm}$ and each stone locates in an appropriate position (pelvis or calyx with an uncomplicated structure of the collection system), patients without ectopia of the ureteral orifice and ureterostenosis can be initially treated by RIRS. If this fails, the operation can then be turned into a mini-PCNL procedure. (II) If the solitary stone or total multiple lithiasis is more than $2 \mathrm{~cm}$ in size or the total of multiple stones is less than $2 \mathrm{~cm}$ with a complicated structure of the collection system, patients without laparoscopic contraindications can be treated by laparoscopy-assisted mini-PCNL. (III) In all other situations, mini-PCNL can be performed without laparoscopy-assistance, and open surgery can be used as a final method to deal with various failures. This study is limited by its small sample size and a larger prospective randomized study is recommended to verify the results.

\section{Conclusions}

Although more time-consuming and invasive than RIRS, mini-PCNL could deal with larger lithiasis and more complex situations with a higher success rate. Both miniPCNL and RIRS are feasible and safe treatments for pelvic ectopic kidney lithiasis with each holding unique advantages. Hence in practice, an appropriate individualized treatment should be selected depending on the individual characteristics of the patients.

\section{Acknowledgments}

Funding: None.

\section{Footnote}

Reporting Checklist: The authors have completed the MDAR reporting checklist. Available at http://dx.doi.org/10.21037/ tau-21-77
Data Sharing Statement: Available at http://dx.doi. org/10.21037/tau-21-77

Conflicts of Interest: Both authors have completed the ICMJE uniform disclosure form (available at http://dx.doi. org/10.21037/tau-21-77). The authors have no conflicts of interest to declare.

Etbical Statement: The authors are accountable for all aspects of the work in ensuring that questions related to the accuracy or integrity of any part of the work are appropriately investigated and resolved. The study conformed to the provisions of the Declaration of Helsinki (as revisedin2013). The study was approved by the medical ethics committee of the Qiandongnan People's Hospital Affiliated to Guizhou Medical University. All patients agreed to participate in this study and signed an informed consent form.

Open Access Statement: This is an Open Access article distributed in accordance with the Creative Commons Attribution-NonCommercial-NoDerivs 4.0 International License (CC BY-NC-ND 4.0), which permits the noncommercial replication and distribution of the article with the strict proviso that no changes or edits are made and the original work is properly cited (including links to both the formal publication through the relevant DOI and the license). See: https://creativecommons.org/licenses/by-nc-nd/4.0/.

\section{References}

1. Zafar FS, Lingeman JE. Value of laparoscopy in the management of calculi complicating renal malformations. J Endourol1996;10:379-83.

2. Tan YK, Cha DY, Gupta M.Management of stones in abnormal situations. Urol Clin North Am 2013;40:79-97.

3. Cinman NM, Okeke Z, Smith AD.Pelvic kidney: associated diseases and treatment. J Endourol2007;21:836-842.

4. Otaño N, Jairath A, Mishra S, et al. Percutaneous nephrolithotomy in pelvic kidneys: is the ultrasoundguided puncture safe? Urology 2015;85:55-8.

5. Yin Z, Wei YB, Liang BL, et al. Initial experiences with laparoscopy and flexible ureteroscopy combination pyeloplasty in management of ectopic pelvic kidney with stone and ureter-pelvic junction obstruction. Urolithiasis 2015;43:255-60.

6. TalicRF.Extracorporeal shock-wave lithotripsy monotherapy in renal pelvic ectopia. Urology 
1996;48:857-61.

7. Demirkesen O, Yaycioglu O, Onal B, et al. Extracorporeal shockwave lithotripsy for stones in abnormal urinary tracts: analysis of results and comparison with normal urinary tracts. J Endourol2001;15:681-5.

8. Bush WH, Brannen GE.Extracorporeal shock-wave lithotripsy (ESWL) of pelvic kidney calculus. Use of C-arm fluoroscopy for correct patient positioning. Urology 1987;29:357-60.

9. Weizer AZ, Springhart WP, Ekeruo WO, et al.Ureteroscopic management of renal calculi in anomalous kidneys. Urology 2005;65:265-9.

10. Zhang W, Zhou T, Wu T, et al. Retrograde Intrarenal Surgery Versus Percutaneous Nephrolithotomy Versus Extracorporeal Shockwave Lithotripsy for Treatment of Lower Pole Renal Stones: A Meta-Analysis and Systematic Review. J Endourol2015;29:745-59.

11. Rao PN, Faulkner K, Sweeney JK, et al. Radiation dose to patient and staff during percutaneous nephrostolithotomy. Br J Urol1987;59:508-12.

12. Karamcheti A, O'Donnell WF. Percutaneous

Cite this article as: $\mathrm{Wu} \mathrm{J}$, Shen $\mathrm{J}$. The safety and efficacy of mini-percutaneous nephrolithotomy vs. retrograde intrarenal surgery for treatment of renal lithiasis in pelvic ectopic kidney: an exploratory pilot study. Transl Androl Urol 2021;10(4):17341742. doi: $10.21037 /$ tau-21-77 nephrolithotomy: an innovative extraction technique. J Urol1977;118:671-2.

13. Agarwal M, Agrawal MS, Jaiswal A, et al. Safety and efficacy of ultrasonography as an adjunct to fluoroscopy for renal access in percutaneous nephrolithotomy (PCNL). BJU Int 2011;108:1346-9.

14. Ganesamoni R, Sabnis RB, Mishra S, et al. Microperc for the management of renal calculi in pelvic ectopic kidneys. Indian J Urol2013;29:257-9.

15. Bozkurt IH, Cirakoglu A, Ozer S. Retroperitoneal laparoscopic pyelolithotomy in an ectopic pelvic kidney. JSLS 2012;16:325-8.

16. D'souza N, Verma A, Rai A. Laparoscopic-assisted mini percutaneous nephrolithotomy in the ectopic pelvic kidney: Outcomes with the laser dusting technique. Urol Ann 2016;8:87-90.

17. Bozkurt OF, Tepeler A, Sninsky B, et al. Flexible ureterorenoscopy for the treatment of kidney stone within pelvic ectopic kidney. Urology 2014;84:1285-9.

(English Language Editor: B. Draper) 